Metodología Docente y Evaluación

\title{
El script concordance test como herramienta evaluativa
}

\author{
Ana María de Santiago Nocito ${ }^{1, *}$ y Alberto García Lledó ${ }^{2}$ \\ 1 Profesor Asociado al Departamento de Medicina y Especialidades Médicas, Universidad de Alcalá; Médico \\ de Familia EAP Cogolludo SESCAM \\ 2 Profesor Asociado al Departamento de Medicina y Especialidades Médicas, Universidad de Alcalá; Jefe de \\ Servicio de Cardiología, Hospital Universitario Príncipe de Asturias, Alcalá de Henares \\ * Autor correspondencia: adesan2@telefonica.net
}

DOI: https://doi.org/10.37536/RIECS.2020.5.2.234

Recibido: 16/11/2020; Aceptado: 27/11/2020; Publicado: 30/11/2020

Resumen: El script concordance test es un instrumento de aprendizaje y de evaluación de las competencias inherentes al razonamiento clínico y a la toma de decisions. La adquisición de experiencia clínica supone la elaboración de redes de conocimiento que se incorporan a la tarea clínica habitual. Estas redes, conocidas como scripts, se organizan para alcanzar metas relativas al diagnóstico, al establecimiento de estrategias de investigación y a opciones de tratamiento. El presente artículo muestra la construcción de este tipo de preguntas y su sistema de evaluación

Palabras Clave: Script concordance test, Razonamiento clínico, Evaluación de competencias.

Abstract: The script concordance test is an instrument for learning and evaluating the inherent competencies in clinical reasoning and decision making. The acquisition of clinical experience implies the elaboration of knowledge networks that are incorporated to the routine clinical tasks. These networks, known as scripts, are organized to achieve goals related to diagnosis, the establishment of research strategies and treatment options. This article shows the construction of this type of questions and its evaluation system

Key words: Script concordance test, Clinical reasoning, Competence evaluation.

\section{1. ¿Qué es un script?}

El script concordance test es un instrumento de aprendizaje y de evaluación de las competencias inherentes al razonamiento clínico y a la toma de decisiones. El término inglés script se puede traducir del castellano "guión" utilizado en las artes escénicas como base interpretativa usada por los actores siguiendo un orden establecido. En informática, un script es un archivo de órdenes, un archivo de procesamiento por lotes, concepto también bien relacionado con este sentido evaluativo.

Cuando se atiende a pacientes reales para resolver un problema se precisa una entrevista detallada en la que se recopilan todos los datos necesarios para formular la demanda y resolverla. La adquisición de experiencia clínica supone la elaboración de redes de conocimiento que se incorporan a la tarea clínica habitual. Estas redes, conocidas como scripts, se organizan para alcanzar metas relativas al diagnóstico, al establecimiento de estrategias de investigación y a opciones de tratamiento. Los scripts son esquemas que comienzan a generarse cuando los estudiantes se enfrentan a sus primeros casos clínicos y van ampliándose y actualizándose durante toda la vida profesional.

Los scripts contienen información acerca de las conexiones que unen los ítems de conocimiento (características clínicas). Estas conexiones que permiten a una persona tomar decisiones con relación a la fuerza o debilidad de una hipótesis, decidir si una característica clínica está asociada o no con tal 
hipótesis, siendo aceptada o rechazada. Por ello, el objetivo del modelo de Script no es medir conocimientos, sino evaluar el proceso de razonamiento clínico, la organización de éste en redes conceptuales y la capacidad de activación de estas redes en la toma de decisiones frente a una situación clínica concreta.

El script concordance test sitúa a los examinados ante situaciones clínicas auténticas en las que tienen que interpretar datos para tomar decisiones. Se puede usar en soporte escrito o informático, y está validado como herramienta evaluadora en el grado, formación especializada y en educación médica continuada.

El sistema de puntuación está diseñado para medir la diferencia que existe entre los scripts del estudiante y de un panel de expertos utilizando una escala de Likert. Esta escala de Likert permite conocer el grado de conformidad del encuestado con las afirmaciones propuestas permitiendo matizar su opinión. El número de ítem más utilizado sea el de 5 niveles, pero también se pueden utilizar 4 ó 7.

\section{2. ¿Cómo se construye un script?}

La construcción del test de concordancia de scripts requiere la colaboración de un pequeño grupo de expertos al que se pide que describan algunas situaciones clínicas representativas de su campo y que susciten cierta incertidumbre.

Para cada situación que plantea un script se debe especificar:

1. Viñeta: Expone una situación clínica real que ha de ser resulta.

2. Una información adicional que muestra posibles hipótesis concordantes con la situación clínica y que el alumno debe de analizar

3. Una evaluación sobre la hipótesis original utilizando una escala de Likert.

La evaluación dada por el alumno se ha de comparar con las respuestas de un grupo de expertos en la materia.

Veamos un ejemplo en la tabla I:

Paciente de 84 años que pone aviso a domicilio porque tiene fiebre y malestar general desde el día anterior.

Tabla I Test de Script de Diagnostico (Modificado de Escobar Rabadán F, López-Torres Hidalgo J. “¿Necesitamos nuevos instrumentos para la evaluación de los residentes de Medicina de Familia?”. Francisco Escobar Rabadán, Jesús López-Torres Hidalgo. Rev Clin Med Fam vol.2 no.6 Albacete feb. 2009)

\begin{tabular}{|l|l|l|l|l|l|l|}
\hline \multicolumn{1}{|c|}{$\begin{array}{c}\text { Si disponemos de la } \\
\text { siguiente información }\end{array}$} & $\begin{array}{l}\text { De cara a considerar la } \\
\text { siguiente } \\
\text { diagnóstica }\end{array}$ & \multicolumn{2}{|l|}{$\begin{array}{l}\text { El efecto de la información } \\
\text { previa sobre su hipótesis } \\
\text { es: }\end{array}$} \\
\hline $\begin{array}{l}\text { Tiene cefalea, dolor lumbar y } \\
\text { accesos de fiebre mayor de } \\
\mathbf{4 0}\end{array}$ & Pielonefritis & -2 & -1 & 0 & 1 & 2 \\
\hline $\begin{array}{l}\text { Es fumador de } 5 \text { cigarrillos } \\
\text { diarios }\end{array}$ & $\begin{array}{l}\text { Agudización de la enfermedad } \\
\text { pulmonar obstructiva crónica }\end{array}$ & & & & & \\
\hline $\begin{array}{l}\text { Tiene herpes labial, esputo } \\
\text { herrumbroso y dolor } \\
\text { inspiratorio }\end{array}$ & Neumonía & & & & & \\
\hline
\end{tabular}




\begin{tabular}{|l|l|l|l|l|l|l|}
\hline $\begin{array}{l}\text { Tiene tos y expectoración } \\
\text { abundantes }\end{array}$ & Bronquiectasias & & & & & \\
\hline $\begin{array}{l}\text { La exploración de faringe es } \\
\text { normal }\end{array}$ & Faringoamigdalitis & & & & & \\
\hline $\begin{array}{l}\text { Hace } 3 \text { días no ha estado con } \\
\text { nadie, es el mes de julio }\end{array}$ & Gripe & & & & & \\
\hline
\end{tabular}

-2: Excluye totalmente o casi totalmente la hipótesis

-1: Hipótesis poco probable 0: No tiene impacto sobre

la hipótesis (efecto neutro)

+1: Hipótesis más probable

+2 : Confirma totalmente o casi con certeza la

hipótesis

\subsection{Características}

1. Las viñetas escriben situaciones clínicas reales y frecuentes seguidas de hipótesis concordantes con la situación clínica.

2. Nuevas informaciones, independientes entre sí, para ayudar a resolver el problema. Puede haber 4, 5, 6...incluso 10 ítems (En este ejemplo: pielonefritis, agudización del EPOC, neumonía...)

3. Para construir los diferentes ítems hay que tener en cuenta que SON INDEPENDIENTES entre sí y que tienen la finalidad de determinar el impacto de la nueva información sobre la hipótesis.

4. Todos los datos para la solución del problema no están presentes en la viñeta. No se trata de resolver el problema, sino de seguir el proceso según los nuevos datos.

5. No existe una única respuesta aceptable. Las respuestas se obtienen de un panel formado por 10 o 20 expertos, que contestan según su experiencia. Puede no haber unanimidad, por lo que pueden considerarse varias respuestas válidas, pero con distinta puntuación.

6. Los scripts pueden tratar cuestiones relativas al diagnóstico, a la realización de pruebas complementarias o al tratamiento.

\section{3. ¿Cómo se puntúa un script?}

Por consenso se otorga un punto por cada ítem acertado. Las respuestas correctas son las que han coincidido mayor número de expertos, esa es la forma técnicamente correcta de evaluar los script.

La otra forma es la de otorgar también puntuación a otras respuestas dadas por expertos, aunque no hayan sido las más aceptadas. Es decir, si coincide con el segundo grupo más votado por los expertos obtiene una puntuación menor a uno. 5

Se aconseja a los alumnos que practiquen en parejas o pequeños grupos estos y otros ejemplos de la práctica diaria, ya que el script evalúa el razonamiento para tomar decisiones y éste debe de ser entrenado durante el proceso de aprendizaje. 


\section{Referencias Bibliográficas}

1. Charlin B, Tardif J, Boshuizn HPA. "Scripts and medical diagnostic knowledge: theory and application for clinical reasoning instruction and research". Acad Med 2000; 75: 182-190

2. "Evaluación del aprendizaje, estrategias innovadoras" (SCRIPT) Dr. Carlos Braylovsky, Director Centre de Evaluation des Sciencies de la Santé (CESSUL), Faculté de Médicine, Université Laval, Québec. Canadá.

3. Francisco Escobar Rabadán, Jesús López-Torres Hidalgo. “¿Necesitamos nuevos instrumentos para la evaluación de los residentes de Medicina de Familia?”. Francisco Escobar Rabadán, Jesús López-Torres Hidalgo. Rev Clin Med Fam vol.2 no.6 Albacete feb. 2009

(C) 2020 por los autores; Esta obra está sujeta a la licencia de Reconocimiento 4.0 Internacional de Creative Commons. Para ver una copia de esta licencia, visite http://creativecommons.org/licenses/by-nc-nd/4.0/. 